\title{
Erratum to: Robust Exponential Decay of Correlations for Singular-Flows
}

\author{
Vítor Araújo ${ }^{1}$, Paulo Varandas ${ }^{2}$ \\ ${ }^{1}$ Instituto de Matemática, Universidade Federal da Bahia, Av. Ademar de Barros s/n, \\ 40170-110 Salvador, Brazil.E-mail: vitor.araujo@pq.cnpq.br \\ 2 Departamento de Matemática, Universidade Federal da Bahia, Av. Ademar de Barros s/n, \\ 40170-110 Salvador, Brazil. E-mail: paulo.varandas@ufba.br
}

Received: 6 November 2014 / Accepted: 24 July 2015

Published online: 19 October 2015 - (C) Springer-Verlag Berlin Heidelberg 2015

Commun. Math Phys. 311, 215-246 (2012)

We correct the proof of the main statement in Sect. 4.2.3 of the original article.

It has been pointed out to the authors by Butterley and Melbourne that the arguments in the original article are not enough to prove the following:

Theorem A. Given any compact 3-manifold $M$, for each $s \geq 2$ there exists $N=N(s) \geq$ 2 and an open subset $\mathcal{U}$ of $\mathfrak{X}^{N}(M)$ such that each $X \in \mathcal{U}$ exhibits a geometric Lorenzflow, which is $C^{S}$-smoothly semi-conjugated to a good hyperbolic skew-product semi-flow.

In this erratum, we show how to complete the proof of Theorem A. Consequently, the main result in the original article remains valid. The need for possible higher smoothness of the vector field to be in a certain conjugation class is a direct consequence of a classical result of Hartman [3, Theorem 12.1, p. 257] stated also in Theorem 3.2 of the original article. Let $s \geq 2$ and let $\mathcal{U}$ be a $C^{N}$-open set of vector fields that exhibit a Lorenz attractor and let $r: \Delta \rightarrow \mathbb{R}^{+}$denote the roof function obtained by conjugation to a suspension flow with a global cross-section $\Delta$.

The good hyperbolic skew-product semiflow condition consists of exponential tail estimates for the roof function, smoothness of the disintegration of the SRB measure and a non-uniform integrability (UNI) condition; see Sect. 2 of the original article. The argument in Sect. 4.4 of the original article to prove smoothness of the disintegration of the SRB measure along stable manifolds lacks some details that were recently completed by Butterley and Melbourne in [2]. Since the last estimate in the argument in Sect. 4.2.3 of the original article is not enough to guarantee the uniform non-integrability condition claimed, the goal of this erratum is to provide an alternative proof to the uniform non-integrability condition. The argument in Sect. 4.2.3 of the original article must 
be replaced by the following result asserting that the non-integrability condition is a $C^{1+}$-open and $C^{k}$-dense condition, for all $N \leq k<\infty$.

Proposition 1. For any $N \leq k<\infty$ and $\varepsilon>0$ there exists a $C^{1+\varepsilon}$-open and $C^{k}$-dense set of vector fields $\mathcal{V} \subset \mathcal{U}$, so that the roof function $r$ of every $X \in \mathcal{V}$ satisfies (UNI): it is not possible to write $r=v+u \circ F-u$ where $v: \Delta \rightarrow \mathbb{R}$ is constant on each $\Delta^{\ell}$ and $u: \Delta \rightarrow \mathbb{R}$ is a $C^{1}$-function.

The remainder of this corrigendum is devoted to the proof of this proposition. For any fixed $N \leq k<\infty$ and $\varepsilon>0$, if there exists a $C^{1}$ function $u: \Delta \rightarrow \mathbb{R}$ and a measurable function $v: \Delta \rightarrow \mathbb{R}$ constant on each element $\omega$ of $\mathcal{Q}$ satisfying $r=u \circ F-u+v$, for geometric Lorenz flow constructed as in the original article with $X \in \mathfrak{X}^{k}(M)$, then we show that, up to a $C^{k}$ arbitrarily small perturbation of the original vector field $X$, this is impossible for all $C^{1+\varepsilon}$ nearby vector fields.

For this we choose two distinct periodic points $x_{1}, x_{2}$ for $F: \Delta \rightarrow \Delta$ of the same period $n$ whose orbits are (i) distinct, and (ii) each orbit visits each of the elements of the Markov partition the same number of times as the other, but (iii) necessarily in some different order to each other. The existence of such a pair of periodic orbits is a consequence of $F$ being a full branch Markov map: if $\omega_{1}, \omega_{2}$ are two elements of the Markov partition, we can choose the period $p=4$ and $x_{i}, i=1,2$ such that

$$
x_{1}, F\left(x_{1}\right) \in \omega_{1}, F^{2}\left(x_{1}\right), F^{3}\left(x_{1}\right) \in \omega_{2} \quad \text { and } \quad x_{2}, F^{2}\left(x_{2}\right) \in \omega_{1}, F\left(x_{2}\right), F^{3}\left(x_{2}\right) \in \omega_{2} .
$$

Furthermore, $x_{1}, x_{2}$ can be chosen in the interior of $\omega_{1}$. The cohomological equation implies $S_{p} r\left(x_{1}\right)=S_{p} v\left(x_{1}\right)=S_{p} v\left(x_{2}\right)=S_{p} r\left(x_{2}\right)$ since $x_{1}$ and $x_{2}$ visit the same Markov partition elements an equal number of times and $v$ is constant on each partition element. Hence it is enough to modify the roof function $\rho: S \rightarrow \mathbb{R}^{+}$in a small neighbourhood of $x_{1}$ that does not intersect the orbit of $x_{2}$ to ensure that the induced roof function $r$ satisfies $S_{p} r\left(x_{1}\right)>S_{p} r\left(x_{2}\right)$ and so is not cohomologous to a piecewise constant roof function. Note that $S_{p} r\left(x_{i}\right)$ is the period of $x_{i}$ as a periodic orbit of the vector field $X$. This modification can be done by changing the size of the vector field $X$ in a small neighborhood around $x_{1}$.

Indeed, let $U_{0}, U_{1}$ be small open neighborhoods of $x_{2}$ that do not intersect the orbit of $x_{1}$ with $U_{0} \subset \overline{U_{0}} \subsetneq U_{1}$ and assume that on $U_{1}$ we have $C^{k}$-linearized the flow via the Tubular Neighborhood Theorem, that is, up to a $C^{k}$ coordinate change, $X$ in $U_{1}$ is constant and given by $e_{1}=(1,0,0)$. Take a $C^{\infty}$ function $\varphi: M \rightarrow[0,1]$ that vanishes in the complement of $U_{1}$ and is constant equal to one in $U_{0}$ and consider $Z=\varphi \cdot e_{1} \in \mathfrak{X}^{\infty}(M)$. Now consider $Y_{\delta}=X+\delta Z \in \mathfrak{X}^{k}(M)$, which is $\delta$ close to $X$ in the $C^{k}$-topology: $Y_{\delta}$ equals $X$ in $M \backslash U_{1}$ and equals (with a slight abuse of notation) $X+\delta e_{1}$ for points in $U_{0}$. For $Y_{\delta}$ both $x_{1}$ and $x_{2}$ are still periodic points, but the period of $x_{2}$ decreases while the period of $x_{1}$ is kept the same. By some abuse of notation we shall denote by $X$ the perturbed vector field.

We need to show that this conclusion holds for all vector fields $Y$ that are $C^{1+\varepsilon}$ close to $X$. The orbits of these points involve only finitely many iterates (the inducing time $R$ ) of the Lorenz transformations $f$ : both orbits are contained in $\{R \leq L\}$ for some fixed $L \geq 1$. Moreover, these points belong to two distinct hyperbolic periodic orbits (of saddle type) of the geometric Lorenz attractor and are away from a neighborhood of the singularity at the origin. Hence, these orbits admit a smooth continuation to all $C^{1+\varepsilon}$ nearby vector fields $Y$ that admit a similar construction of smooth cross-section $S_{Y}$ and induced transformation $F_{Y}$, following the inductive procedure detailed in [1]. 
In particular, since the periodic points $x_{1}, x_{2}$ belong to the interior of the partition elements, we can control finitely many iterates of the Lorenz transformation $f_{Y}$ and obtain that the corresponding partition of $\left\{R_{Y} \leq L\right\}$ associated to the induced transformation $\tilde{F}$, up to the inducing time $L$, is close enough to the partition of $\{R \leq L\}$ so that the continuation $\tilde{x}_{i}$ of the orbits of $x_{i}, i=1,2$ have the same combinatorics as before, visiting the same elements of the Markov partition the same number of times as the other but in a different order, and with the same inducing times. In this argument it is crucial that all the ingredients in the inductive construction be preserved for $Y$. In particular, the size of hyperbolic balls obtained from hyperbolic times of the one-dimensional Lorenz transformation $f_{Y}$, which depend on the rates of expansion of $f_{Y}$ but also on the Hölder exponent of $D f_{Y}$ (see [1]). In its turn, the Hölder exponent of $D f_{Y}$ depends on the smoothness of the strong-stable foliation of the Lorenz attractor. Hence, the procedure described here demands that the vector fields $Y$ and $X$ are at least $C^{1+\varepsilon}$-close.

The rest of the inducing map for $f_{Y}$ is obtained following an inductive construction and we do not use in this argument the elements of the partition whose induction time is higher than $L$.

We can then use the same expression as before obtaining $S_{p} \tilde{r}\left(\tilde{x}_{1}\right)>S_{p} \tilde{r}\left(\tilde{x}_{2}\right)$ for $Y$ sufficiently $C^{1+\varepsilon}$ close to $X$, showing that the induced roof function $\tilde{r}$ for the vector field $Y$ cannot be cohomologous to a piecewise constant roof function.

This completes the proof of the proposition by proving that this function satisfies the uniform non-integrability condition needed to obtain exponential decay of correlations for the flow of $Y$ on the geometric Lorenz attractor.

\section{References}

1. Alves, J.F., Luzzatto, S., Pinheiro, V.: Lyapunov exponents and rates of mixing for one-dimensional maps. Ergod. Theory Dyn. Syst. 24(3), 637-657 (2004)

2. Butterley, O., Melbourne, I.: Disintegration of invariant measures for hyperbolic skew products. Isr J Math (to appear). arXiv:1503.04319

3. Hartman, P.: Ordinary Differential Equations. Classics in Applied Mathematics, vol. 38. Society for Industrial and Applied Mathematics (SIAM), Philadelphia (2002). (Corrected reprint of the second (1982) edition [Birkhäuser, Boston, MA; MR0658490 (83e:34002)], with a foreword by Peter Bates.) 